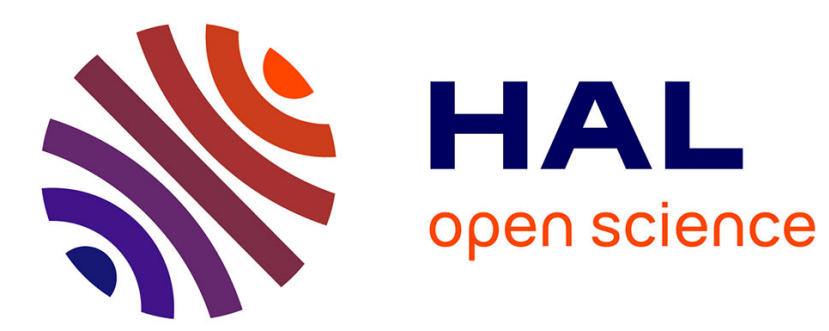

\title{
Retraction obstruction to time-varying stabilization
}

Emmanuel Bernuau, Wilfrid Perruquetti, Emmanuel Moulay

\section{To cite this version:}

Emmanuel Bernuau, Wilfrid Perruquetti, Emmanuel Moulay. Retraction obstruction to time-varying stabilization. Automatica, 2013, 49 (6), pp.1941-1943. 10.1016/j.automatica.2013.03.012 . hal00862934

\section{HAL Id: hal-00862934 \\ https://hal.science/hal-00862934}

Submitted on 19 Jan 2021

HAL is a multi-disciplinary open access archive for the deposit and dissemination of scientific research documents, whether they are published or not. The documents may come from teaching and research institutions in France or abroad, or from public or private research centers.
L'archive ouverte pluridisciplinaire HAL, est destinée au dépôt et à la diffusion de documents scientifiques de niveau recherche, publiés ou non, émanant des établissements d'enseignement et de recherche français ou étrangers, des laboratoires publics ou privés. 


\title{
Retraction obstruction to time-varying stabilization
}

\author{
Emmanuel Bernuau ${ }^{a}$, Wilfrid Perruquetti ${ }^{\mathrm{a}, \mathrm{c}}$, Emmanuel Moulay ${ }^{\mathrm{b}}$ \\ ${ }^{\mathrm{a}} L A G I S$ (UMR-CNRS 8219) \\ Ecole Centrale de Lille, BP 48 \\ 59651 Villeneuve D'Ascq, France \\ ${ }^{\mathrm{b}}$ Xlim (UMR-CNRS 7252) - Département SIC \\ Université de Poitiers - Bât. SP2MI, Bvd Marie et Pierre Curie - BP 30179 \\ 86962 Futuroscope Chasseneuil Cedex, France \\ ${ }^{\mathrm{c}}$ Non-A project at INRIA Lille - Nord Europe \\ Parc Scientifique de la Haute Borne, 40 avenue Halley, Bt.A Park Plaza \\ 59650 Villeneuve dAscq, France
}

\begin{abstract}
This paper address the problem of the global stabilization on a total space of a fiber bundle with a compact base space. We prove that, under mild assumptions (existence of a continuous section and forward unicity of solutions), no equilibrium of a continuous system defined on such a state space can be globally asymptotically uniformly stabilized using continuous time-varying feedback.
\end{abstract}

Key words: Retraction obstruction, Lyapunov stability, stabilization.

\section{Introduction}

Topological obstruction to stabilization is a long standing problem of control theory detailed in the introduction of [1]. There are two main topological obstructions to continuous stabilization. First, the Brockett obstruction is a local obstruction related to the structure of the controlled systems involving the nature of feedback controls [2]. Then, the retraction obstruction is a global obstruction related to the structure of the underlying state space: if the state space of the system has the structure of a vector bundle over a compact manifold, no continuous static feedback can globally stabilize an equilibrium. This result has been proved and its consequences studied in detail in [3].

The Brockett condition, which is necessary in the case of continuous time-invariant feedback controls, does not remain necessary for driftless controllable systems with time-varying feedback. The existence of such feedbacks has been proved in [4], while [5] gave an explicit de-

Email addresses: emmanuel.bernuau@ec-lille.fr (Emmanuel Bernuau), wilfrid.perruquetti@inria.fr (Wilfrid Perruquetti),

emmanuel.moulay@univ-poitiers.fr (Emmanuel Moulay). sign under an additional condition on the Control Lie Algebra (see [5, Assumption 1]). A natural question is, hence, to wonder whether continuous time-varying feedback controls could also avoid the retraction obstruction as suggested in the introduction of [6].

In $[3$, Remark 1], the authors mention that their result also handle the case of dynamic feedback. Indeed a dynamic feedback is usually seen as a dynamic extension where the augmented state is stable. Estimation of parameters or observer-based control are in this scope. In that case, the result of [3] is applicable directly, with the method exposed in their remark. Nevertheless, a timevarying static feedback is also a dynamic extension using a timer $\dot{\tau}=1$. However in this situation, there is no convergence to a single equilibrium point, but to a submanifold; the result of [3] is therefore not applicable in this context, following [3, Remark 1].

The aim of this paper is to prove that, in the second case, the obstruction still remains: a time-varying feedback control which is globally asymptotically uniformly stabilizing a system defined on a fiber bundle with a compact manifold as its base space does not exist. 


\section{Retraction obstruction}

By a manifold we mean a smooth, positive dimensional, connected manifold without boundary. The definition of a fiber bundle is given for instance in [7].

Our purpose is to link the topological property of contractibility of a manifold to the existence of a globally asymptotically stable equilibrium. Let us introduce the definitions we will be using and some useful properties.

Definition 1 Let $E$ be a topological space and $x_{0} \in E$. $A$ retraction of $E$ on $x_{0}$ is a continuous mapping $h$ : $[0,1] \times E \rightarrow E$ such that for all $x \in E, h(0, x)=x$ and $h(1, x)=x_{0}$. A topological space $E$ is said to be contractible if there exists a retraction of $E$.

Proposition 2 [8, Section 2.4] No compact manifold is contractible.

Consider a controlled system defined by

$$
\dot{y}=f_{0}(y, u) \quad y \in \mathcal{N}, u \in \mathrm{U}
$$

with $\mathcal{N}$ a manifold and $U$ a set of admissible controls, and where $f_{0}$ is a continuous vector field.

We wonder about the global stabilizability of the system via a time-varying feedback $u(y, t)$. To do that, we will set $\dot{\tau}=1$ and look at the partial asymptotic stability of the closed-loop system:

$$
\begin{aligned}
& \dot{y}=f_{0}(y, u(y, \tau)) \\
& \dot{\tau}=1
\end{aligned} .
$$

Hence, let us introduce the definitions of partial stability we will be using. Those definitions are adapted from [9].

Definition 3 Let $\mathcal{M}=\mathcal{N} \times \mathcal{T}$ be a product manifold. Consider $f=\left(f_{1}, f_{2}\right)$ a forward complete continuous vector field on $\mathcal{M}$ with the property of unicity of solutions in forward time. We denote by $\Phi$ the semiflow of $f$ and $p_{1}$ the canonical projection on $\mathcal{N}$.

(1) We say that $y_{\infty} \in \mathcal{N}$ is a partial equilibrium if for all $\tau \in \mathcal{T}$, we have $f_{1}\left(y_{\infty}, \tau\right)=0$.

(2) A partial equilibrium $y_{\infty} \in \mathcal{N}$ is said to be partially stable uniformly in $\tau$ if for all $U \subset \mathcal{N}$ neighborhood of $y_{\infty}$ there exists $V \subset \mathcal{N}$ a neighborhood of $y_{\infty}$ such that for all $y \in V$ and for all $\tau \in \mathcal{T}, p_{1} \circ \Phi(t,(y, \tau)) \in$ $U$ for all $t \geq 0$.

(3) A partial equilibrium $y_{\infty} \in \mathcal{N}$ is said to be partially globally asymptotically stable uniformly in $\tau$ if it is partially stable uniformly in $\tau$ and if for all $(y, \tau) \in$ $\mathcal{M}$ we have $p_{1} \circ \Phi(t,(y, \tau)) \rightarrow y_{\infty}$ when $t \rightarrow+\infty$.
Remark 4 The last item of definition 3 is slightly different from the more standard ones. Indeed, to prove our result, we only need the stability to be uniform with respect to $\tau$. The uniformity with respect to $\tau$ of the convergence, which is usually required, is not necessary here.

The following definition, inspired by [10, Chapter 12] about time-varying stabilizability, is here given in the partial stability context.

Definition 5 The system (1) is said to be globally asymptotically uniformly stabilizable by means of a continuous generalized time-varying feedback if there exist a point $y_{\infty} \in \mathcal{N}$, a manifold $\mathcal{T}$, a continuous mapping $f_{2}: \mathcal{N} \times \mathcal{T} \rightarrow \mathrm{T} \mathcal{T}$ with $f_{2}(y, \tau) \in \mathrm{T}_{\tau} \mathcal{T}$ for all $y \in \mathcal{N}$ and all $\tau \in \mathcal{T}$ and a continuous control law $u(y, \tau)$ such that $y_{\infty}$ is a partial equilibrium of the closed loop system $(\dot{y}, \dot{\tau})=\left(f_{0}(y, u(y, \tau)), f_{2}(y, \tau)\right)$ and is partially globally asymptotically stable uniformly in $\tau$.

Remark 6 Let us note that, taking $\mathcal{T}=\mathbb{R}$ and $f_{2}(y, \tau)=1$, this definition boils down to the definition of global asymptotic stabilization by means of a continuous time-varying feedback. In the generalized time-varying setting, the variable $\tau$ can be stable or not, scalar or vector, bounded or not.

In [3, Theorem 1], the authors use Proposition 2 to prove that if a manifold $\mathcal{N}$ admits a structure of fiber bundle over a compact manifold, then no continuous vector field over $\mathcal{N}$ can have an unique globally asymptotically stable equilibrium. Hence, they conclude that the system (1) cannot be globally asymptotically stabilized by means of a state feedback. Let us prove now that [3, Theorem 1] can be extended in the following way to the time-varying setting.

Theorem 7 Assume that $\mathcal{N}$ is a manifold with a structure of fiber bundle over a compact manifold $\mathcal{Q}$. If there exists a continuous section of the bundle, then the system (1) is not globally asymptotically uniformly stabilizable by means of a continuous generalized time-varying feedback in such a way that the augmented vector field has the forward unicity of solutions property.

Proof. Ad absurdum, assume that there exists a continuous dynamic feedback which globally asymptotically uniformly stabilizes the system (1) in such a way that the augmented vector field has the forward unicity of solutions. Let us denote by $\tau$ the added variable, and $\tau \in \mathcal{T}$. We have $\dot{\tau}=f_{2}(y, \tau)$, and the system (1) can be rewritten in an extended form, with $f_{1}(y, \tau)=f_{0}(y, u(y, \tau))$ :

$$
\left(\begin{array}{c}
\dot{y} \\
\dot{\tau}
\end{array}\right)=\left(\begin{array}{l}
f_{1}(y, \tau) \\
f_{2}(y, \tau)
\end{array}\right) .
$$

The equation (3) defines a continuous vector field $f$ on the manifold $\mathcal{M}=\mathcal{N} \times \mathcal{T}$, with the forward unicity 
of solutions property. Moreover, there exists a partially globally asymptotically stable equilibrium uniformly in $\tau$ denoted by $y_{\infty} \in \mathcal{N}$.

Let us denote $\pi_{0}: \mathcal{N} \rightarrow \mathcal{Q}$ the fiber bundle projection. Set $p_{1}: \mathcal{M} \rightarrow \mathcal{N}$ the first canonical projection and set $\pi=\pi_{0} \circ p_{1}$. We denote $q_{\infty}=\pi_{0}\left(y_{\infty}\right)$. Similarly, fix $\tau_{0}$ in $\mathcal{T}$ and set $\sigma(q)=\left(\sigma_{0}(q), \tau_{0}\right)$ where $\sigma_{0}: \mathcal{Q} \rightarrow \mathcal{N}$ is a continuous section of $\pi_{0}$. Clearly, $\sigma$ is a continuous section of $\pi$. We also note that the manifold $\mathcal{M}$ trivially inherits a structure of fiber bundle over $\mathcal{Q}$ with projection $\pi$. The vector field $f$ is continuous and has the forward unicity of solutions property. Therefore, it admits a semiflow $\Phi[11]$. Let us denote:

$$
\begin{aligned}
h:[0,1] \times \mathcal{Q} & \rightarrow \mathcal{Q} \\
(\lambda, q) & \mapsto \begin{cases}\pi \circ \Phi\left(\ln \left(\frac{1}{1-\lambda}\right), \sigma(q)\right) & \text { if } \lambda \neq 1 \\
q_{\infty} & \text { if } \lambda=1\end{cases}
\end{aligned}
$$

Since we clearly have $h(0, q)=q$ and $h(1, q)=q_{\infty}$, let us prove the continuity of $h$. This mapping is obviously continuous on $[0,1) \times \mathcal{Q}$.

Let us show the continuity at $(1, q)$ for $q \in \mathcal{Q}$. Let $\left(\lambda_{n}, q_{n}\right) \in[0,1) \times \mathcal{Q}$ be a sequence of points converging to $(1, q)$. We set

$$
t_{n}=\ln \left(\frac{1}{1-\lambda_{n}}\right), x_{n}=\sigma\left(q_{n}\right), x=\sigma(q)
$$

We have $t_{n} \rightarrow+\infty$ and, by continuity of the section, $x_{n} \rightarrow x$. Let $U \subset \mathcal{Q}$ be a neighborhood of $q_{\infty}$ and $U_{0}=\pi_{0}^{-1}(U) \subset \mathcal{N}$ the corresponding neighborhood of $y_{\infty}$. By partial stability uniformly in $\tau$, there exists $V_{0} \subset \mathcal{N}$ a neighborhood of $y_{\infty}$ such that for all $y \in V_{0}$, all $\tau \in \mathcal{T}$ and all $t \geq 0$, we have $p_{1} \circ \Phi(t,(y, \tau)) \subset U_{0}$. On the other hand, the partial attractivity of $y_{\infty}$ means that $p_{1} \circ \Phi(t, x) \rightarrow y_{\infty}$ when $t \rightarrow \infty$. Thus, there exists $T>0$ such that $p_{1} \circ \Phi(T, x) \in V_{0}$. By continuity, there exists $N_{1}>0$ such that for all $n>N_{1}$ we have $p_{1} \circ \Phi\left(T, x_{n}\right) \in V_{0}$. Therefore, for all $t \geq T$, we have $p_{1} \circ \Phi\left(t, x_{n}\right) \in U_{0}$. But $t_{n} \rightarrow+\infty$, so there exists $N_{2}>0$ such that for all $n>N_{2}, t_{n}>T$. Thus, for all $n>N=\max \left(N_{1}, N_{2}\right)$, we have $p_{1} \circ \Phi\left(t_{n}, x_{n}\right) \in U_{0}$. Hence, for all $n>N, h\left(\lambda_{n}, q_{n}\right)=\pi_{0} \circ p_{1} \circ \Phi\left(t_{n}, x_{n}\right) \in U$. Since $U$ is an arbitrary neighborhood of $q_{\infty}$, the mapping $h$ is continuous.

However, the mapping $h$ defines a retraction of the compact manifold $\mathcal{Q}$ on $q_{\infty}$, which leads to the expected contradiction thanks to Proposition 2.

Example 8 Let us consider the following system, defined on the circle:

$$
\ddot{\theta}=u, \quad \theta \in \mathbb{S}^{1} .
$$

By using the angular velocity $\omega=\dot{\theta}$ we can rewrite the system as

$$
\left\{\begin{array}{l}
\dot{\theta}=\omega \\
\dot{\omega}=u
\end{array} .\right.
$$

Here the state space is the tangent space of the circle, denoted by $\mathrm{TS}^{1}$. The tangent space of a manifold has always a structure of vector bundle over that manifold, and therefore $\mathrm{TS}^{1}$ has a structure of vector bundle over the compact manifold $\mathbb{S}^{1}$ with projection $\pi_{0}$ given by:

$$
\pi_{0}(\theta, \omega)=\theta
$$

Moreover, being a vector bundle, the tangent bundle admits $\sigma_{0}$, the zero section, as a continuous section. One may wonder if it is possible to design a continuous feedback control $u(\theta, \omega, t)$ globally stabilizing a state $\left(\theta_{0}, 0\right)$ such that the closed-loop system has uniqueness of solution in forward time.

Since $\mathbb{S}^{1}$ is compact, from Theorem 7 the system (5) cannot be globally asymptotically uniformly stabilized.

Finally, taking into account Theorem 7 and [3, Theorem $1]$, we have the following result: consider the system (1) defined on a manifold with a structure of fiber bundle over a compact manifold. If there exists a continuous section of the bundle, then no continuous dynamic feedback can globally asymptotically uniformly stabilize the system in such a way that the closed loop system has the forward unicity of solutions.

\section{Conclusion}

This paper extends [3, Theorem 1] to the case of timevarying feedback control. We prove that under mild assumptions, no continuous time-varying feedback control can avoid the retraction obstruction; that is, no continuous time-varying feedback control can globally asymptotically uniformly stabilize an equilibrium on a state space which has a structure of fiber bundle over a compact manifold.

This topological obstruction on compact manifolds prevents us from having continuous globally asymptotically uniformly stabilizing feedback (neither static nor time-varying nor dynamic). Moreover it is proved in [12] that the obstruction still remains for discontinuous autonomous vector fields or differential inclusions. Finally the topological obstruction appears to be a strong constraint on stabilization, and few possibilities remain. First, since the uniformity property of the partial stability is indeed being used in our proof, the possibility of non-uniform global stabilizability still remains open. Second, hybrid feedbacks can be considered as suggested in [12]. Finally, other notions of solutions for discontinuous systems exist; some of them might not inherit the same obstruction to global stabilization. 


\section{Acknowledgement}

We would also like to thank the reviewers for their help in the improvement of the paper.

\section{References}

[1] E. Moulay, Q. Hui, Conley index condition for asymptotic stability, Nonlinear Analysis: Theory, Methods \& Applications 74 (13) (2011) 4503-4510.

[2] R. Brockett, Asymptotic stability and feedback stabilization, in "Differential Geometric Control Theory", Birkhäuser, Boston, 1983.

[3] S. P. Bhat, D. S. Bernstein, A topological obstruction to continuous global stabilization of rotational motion and the unwinding phenomenon, Systems \& control letters 39 (1) (2000) 63-70.

[4] J. M. Coron, Global asymptotic stabilization for controllable systems without drift, Math. Control Signals Sytems 5 (3) (1992) 295-312.

[5] J. B. Pomet, Explicit design of time-varying stabilizing control laws for a class of controllable systems without drift, System \& Control Letters 18 (2) (1992) 147-158.

[6] H. Nakamura, Y. Yamashita, H. Nishitani, Minimum projection method for nonsmooth control lyapunov function design on general manifolds, System \& Control Letters 58 (2009) 716-723.

[7] R. Abraham, J. E. Marsden, Foundations of Mechanics, American Mathematical Society, 2008.

[8] V. Guillemin, A. Pollack, Differential topology, American Mathematical Society, 2010.

[9] W. M. Haddad, V. Chellaboina, Nonlinear dynamical systems and control - A Lyapunov based approach, Princeton University Press, 2007.

[10] H. K. Khalil, Nonlinear Systems, 3rd Edition, Prentice Hall, 2002.

[11] N. Bhatia, O. Hajek, Local semi-dynamical systems, Lecture Notes in Mathematics, vol. 90, Springer, 1969.

[12] C. G. Mayhew, A. R. Teel, On the topological structure of attraction basins for differential inclusions, Systems \& Control Letters 60 (2011) 1045-1050. 